\title{
Extraplanar gas and magnetic fields in the cluster spiral galaxy NGC 4569
}

\author{
S. Ryś ${ }^{1}$, K. T. Chyży ${ }^{1}$, M. Weżgowiec ${ }^{1}$, M. Ehle ${ }^{2}$ and R. Beck ${ }^{3}$ \\ ${ }^{1}$ Astronomical Observatory, Jagiellonian University, Kraków, Poland \\ ${ }^{2}$ ESAC, XMM-Newton Science Operations Centre, Madrid, Spain \\ ${ }^{3}$ Max-Planck-Institut für Radioastronomie, Bonn, Germany
}

\begin{abstract}
The Virgo Cluster spiral NGC 4569 is known for its compact starburst in the core and unusual outflow of $\mathrm{H} \alpha$ emitting gas perpendicular to the galaxy disk. Recent radio polarimetric observations with the Effelsberg telescope reveal huge magnetized outflows. Preliminary results of our XMM-Newton observations uncover not only hot gas in the disk but also an extensive X-ray envelope around it. We investigate the possibility of starburst-induced galactic outflows in various gas phases and cluster influence on the galaxy evolution.
\end{abstract}

NGC 4569 is a bright spiral (Sb) galaxy located only $0.5 \mathrm{Mpc}$ from the Virgo Cluster center, known for its compact starburst in the core and a giant $(8 \mathrm{kpc})$ outflow of $\mathrm{H} \alpha$ emitting gas perpendicular to the galaxy disk. Our recent polarimetric radio continuum observations with the Effelsberg telescope at $4.85 \mathrm{GHz}$ and $8.35 \mathrm{GHz}$ reveal huge magnetized lobes, even extending $24 \mathrm{kpc}$ from the galactic plane. This is the first time that such huge radio continuum lobes are observed in a cluster spiral galaxy.

Observing the galaxy in X-rays with XMM-Newton revealed a soft (0.2-1 keV) emission component covering the whole galaxy disk and forming an extensive X-ray envelope around it. In contrast to the radio emission the X-rays do not show similarly large extensions on both sides of the galactic disk. However, stronger X-ray emission is visible close to the disk on its western part, and corresponds to the enhanced radio and $\mathrm{H} \alpha$ emission there. The extension is broad, thus more typical for a wide-spread starburst than for a more collimated ionisation cone from an AGN. The less extended X-ray soft component is also visible to the SW direction from the disk.

The inspection of radio emission from the galaxy lobes indicates that indeed the lobes cannot be powered by an AGN but are probably caused by a nuclear starburst and superwind-type outflows which occurred $\sim 30$ Myr ago. This is supported by estimates of the combined magnetic and cosmic-ray pressure inside the lobes from our radio data. The $\mathrm{H} \alpha$ spur and associated soft X-ray emission on the western part of the disk could be a recent example of such numerous events in the past.

As the galaxy is highly deficient in HI gas it probably suffered from strong ISM-ICM interactions in the form of severe gas stripping. The HI distribution and velocity field suggest that the galaxy is moving at high speed through the ICM in north-east direction. Our X-rays observations showing weaker soft emission in the northern disk and the extension to the south-west support this ram pressure scenario. The large extent of the extraplanar ionized and magnetized gas was probably enabled by the relatively weak ISM density in the heavily stripped galaxy disk.

Acknowledgements. This work was supported by the Polish Ministry of Science and Higher Education, grant 2693/H03/2006/31. Based on observations with the 100-m telescope of the MPIfR at Effelsberg and with XMM-Newton, an ESA science mission with instruments and contributions directly funded by ESA Member States and NASA. 